\title{
Action information from classification learning
}

\author{
Brian H. Ross, Ranyiao Frances Wang, Arthur F. Kramer, \\ Daniel J. Simons, and James A. Crowell \\ University of Illinois at Urbana-Champaign, Urbana, Illinois
}

\begin{abstract}
Much of our learning comes from interacting with objects. Two experiments investigated whether or not arbitrary actions used during category learning with objects might be incorporated into object representations and influence later recognition judgments. In a virtual-reality chamber, participants used distinct arm movements to make different classification responses. During a recognition test phase, these same objects required arm movements that were consistent or inconsistent with the classification movement. In both experiments, consistent movements were facilitated relative to inconsistent movements, suggesting that arbitrary action information is incorporated into the representations.
\end{abstract}

Category learning research has focused on how people learn the features and relations used to categorize new items as members of the category. However, our representations of well-learned items might include both the features of the items themselves and the sensorimotor aspects of our interaction with those objects, such as our actions. Afforded actions do appear to be included in these representations and can influence actions to the items (see below). However, it is not clear whether the affordances are represented or whether they are perceived along with the object. This paper investigates whether even arbitrary actions associated with items during early category learning are incorporated into the item's representation, thereby influencing later actions. Two distinct lines of inquiry suggest that they might be.

First, how we interact with category members influences what we learn about the category (Love, 2003; Markman \& Ross, 2003; Ross, 1996). Category learning research typically focuses on classification - "which category is this item in?"- - but classification per se usually is not the goal. Rather, it is a means toward reaching a goal; classification often supports other goal-related tasks, such as inference or problem solving (Ross, ChinParker, \& Diaz, 2005). Thus, non-classification-related information may be acquired during category learning and accessed for a variety of later tasks. For example, the actions made during the learning of a new category can influence the representation of that category (Smith, 2005): Young children who moved an item horizontally (or vertically) while learning its name were more likely to extend the name to new items that were elongated horizontally (or vertically).

Second, actions involving well-learned items often are associated with those items, especially when the actions are afforded by the objects. Visual recognition of objects automatically activates actions commonly associ- ated with those objects (Creem \& Proffitt, 2001; Pavese \& Buxbaum, 2002; Tucker \& Ellis, 2001). For example, responses are faster to decide if an object (e.g., strawberry, banana) is natural or manufactured if the response is similar to the action for holding that object (precision or power grip, respectively). Chainay and Humphreys (2002) argued that there is a privileged access to action knowledge for objects that relies upon learned associations between seeing these objects and actions. Deciding whether an item could be poured or twisted took less time when people viewed the object's picture than when they read its name, though this was not true for a nonaction semantic decision. Common brain regions also are activated by object-related actions and object identification (e.g., meta-analysis of Grèzes \& Decety, 2001). Even understanding simple sentences (e.g., "John gave me the book") might involve activating some actionrelated information - an action compatibility effect is observed with faster responses when the response action is consistent with the action implied in the sentence (toward the participant, as "gave me" implies) than when it is inconsistent (away from the participant; Glenberg \& Kaschak, 2002). The importance of action has also been highlighted by research on embodied cognition (Barsalou, 1999, 2003; Pecher \& Zwaan, 2005).

Our goal was to investigate whether arbitrary actions associated with items during category learning are incorporated into representations of category members and they influence later actions for those items. Earlier work has used well-learned items with clear affordances to show that knowledge of these affordances is activated during identification. To better examine the learning of associations between actions and objects, we asked people to classify new objects into new categories, and we used a classification response that required an arbitrary action rather than one afforded by the objects.

B. H. Ross, bhross@uiuc.edu 


\section{EXPERIMENT 1}

Category learning consisted of classifying objects and then moving them to the appropriate category bin, using distinctive arm movements within a virtual reality chamber. Following this classification phase, learners were shown old and new objects and had to make old/new recognition responses. For half of the "old" objects, this recognition action was the same one that had been required during classification, whereas for the other "old" objects it was a different action. If arbitrary actions are incorporated into representations during learning, recognition performance should be facilitated (more accurate and/or faster) when the response action is consistent with the action used during learning than when it is inconsistent.

\section{Method}

Participants. Twenty-three undergraduates at the University of Illinois participated in the experiment. The data of one participant were excluded because of system failure, and those of another participant were excluded because the classification learning took too long to finish the experiment.

Design. A classification learning phase was followed by a speeded recognition memory phase. The main manipulation was whether the action during recognition for an item was consistent or inconsistent with the action during classification (within-participant). If the action had been incorporated into the item's representation during category learning, consistent recognition responses should have been more accurate or faster. The particular items that had consistent or inconsistent actions were counterbalanced across participants by switching the locations of the category bins during classification learning.

Apparatus. The experiment was conducted in the Beckman Institute Virtual Reality Cube (www.isl.uiuc.edu/Labs/room_b650 $. \mathrm{htm})$. The Cube is a room ( $3 \mathrm{~m}$ on each side) that allows rearprojection onto all six surfaces via inhouse software. An electromagnetic tracking system (Ascension MotionStar Wireless) measures the position and orientation of the observer, allowing presentations of objects that are rendered correctly from the observer's perspective. An Intel wireless gamepad allows the observer to interact with the virtual objects.

Stimuli. The items were colored geometric figures. A black pattern covered about half their surface area. Each object was of roughly equal size, approximately $930 \mathrm{~cm}^{2}$, and had some depth, as if it had been made by a cookie cutter. For classification learning, the items consisted of three colors, yellow, light blue, and purple; three shapes, semicircle, rectangle, and trapezoid; and three black patterns, stars, triangles, and spots. ${ }^{1}$ One category had items that were yellow or semicircular or both (with any of the three patterns), and the other category's items were light blue or rectangular or both. The purple and trapezoid values were used for both categories. Thus, each category included nine items; for example, the yellow/semicircle category had three yellow trapezoids (one with each of the three patterns), three purple semicircles, and three yellow semicircles.

To increase the number of observations, the recognition test included three blocks, each consisting of these 18 old items and 18 new items. These new items were formed by changing an old item's value on one feature to one of six new values (i.e., there were six new shapes, six new colors, and six new patterns). All items had at least one category-relevant feature (yellow or light blue; or semicircle or rectangle), and each new item was presented only once during the experiment.

Procedure. Each participant was tested individually, and the virtual reality system was calibrated using a measure of their eye spacing. In the classification learning phase, the participant stood on a green cross $2.44 \mathrm{~m}$ from the front wall and looked at a fixation point on that wall made up of two cones with their points touching at the middle (each cone had $25-\mathrm{cm}$ sides and ended in a circle $8 \mathrm{~cm}$ in diameter). When the participant looked at and oriented the gamepad toward that point, a virtual rod extending from the gamepad to the point turned bright white, indicating that the trial could begin. The participant pressed a gamepad button to display and "grab" the object, then moved the rod toward one of two bins positioned against either side wall, one labeled $A$ and the other $B$. As the rod moved, the object moved with it, and when the object was positioned above the bin, it disappeared. Thus, each item was associated with the leftward or rightward action required to place the item in its bin. If the object were placed in the wrong bin, it would move across the front wall to the correct bin. This classification-learning phase continued through a minimum of three blocks until the participant had two blocks (36 trials) with all correct classifications.

The recognition test phase was preceded by 10 practice trials to learn the responses. The two bins were relabeled OLD and NEW. The fixation point was changed into two orthogonal overlapping rectangles (97 cm long and $33 \mathrm{~cm}$ wide) to distinguish the test phase from the learning phase. Participants looked at and oriented the gamepad toward the fixation point to get a bright line, and pressed a key on the gamepad to start the trial. An item then appeared at fixation. For the practice trials, the item was the word old or new, and the task was to move the word to the appropriate bin. No feedback was given.

The speeded recognition task consisted of three blocks of 36 trials, presented without a break between blocks. Each trial was the same as in the practice phase, except that a colored geometric figure with a pattern was shown. Participants were asked to respond quickly while maintaining high accuracy.

\section{Results and Discussion}

Response time (RT) and accuracy were measured both for the initial movement and the final action. The initial movement was defined as the first time the item crossed either of a pair of lines $30 \mathrm{~cm}$ to either side of the fixation point. The final action was when the item was put into a bin. All RTs were trimmed to within two standard deviations of the participant's overall mean (3.6\% of initial RT, $3.9 \%$ of final RT). Only correct responses were included in the RT calculations. All tests used $p<.05$, and effect sizes ( $d$; Cohen, 1988) are given.

Classification learning. The 21 participants included in the analyses all learned to classify the items, though they varied in the number of 18 -item blocks needed to reach the criterion, 3 to $17(M=5.8, S D=3.1)$.

Recognition test. Recognition performance showed good old/new discriminability, with overall accuracy of .834 for initial actions (.806 hits, .862 correct rejections) and .887 for final actions (.864 hits, .909 correct rejections). There was a large action consistency effect for both initial and final accuracy and a small, variable, and nonsignificant RT advantage (see Table 1). The consistent condition was .074 better for initial accuracy $[t(20)=$ $4.14, d=1.28]$ and .071 better for final accuracy $[t(20)=$ $6.04, d=1.86]$. The consistent condition was more accurate for all blocks, but the difference was not always reliable [for initial action, $t(20)=2.36,1.89$, and 2.03 for the three blocks, with corresponding $d \mathrm{~s}$ of $0.73,0.58$, and 0.63 ; for final action, $t(20)=2.22,3.99$, and 1.94, with corresponding $d \mathrm{~s}$ of $0.69,1.23$, and 0.60 ].

Both the initial and final RTs were $21 \mathrm{msec}$ faster in the consistent condition, but neither difference was significant $[t(20)=1.36$ and $0.85, d s$ of 0.42 and 0.26$]$. The initial RT consistency advantage ranged from $48 \mathrm{msec}$ in the 
Table 1

Experiment 1: Mean Response Times (RTs, in Milliseconds) and Proportions Correct for Initial and Final Responses for "Old" Items

\begin{tabular}{cccc}
\hline & Inconsistent & Consistent & $\begin{array}{c}\text { Consistent } \\
\text { Advantage }\end{array}$ \\
\hline Initial RT & & & \\
Overall & 937 & 916 & 21 \\
Block 1 & 935 & 888 & 48 \\
Block 2 & 936 & 921 & 15 \\
Block 3 & 937 & 944 & -7 \\
Initial Accuracy & & & \\
Overall & .769 & .843 & .074 \\
Block 1 & .820 & .905 & .085 \\
Block 2 & .772 & .841 & .069 \\
Block 3 & .714 & .783 & .069 \\
Final RT & & & \\
Overall & 1,265 & 1,244 & 21 \\
Block 1 & 1,259 & 1,213 & 46 \\
Block 2 & 1,237 & 1,241 & -4 \\
Block 3 & 1,290 & 1,277 & 13 \\
Final Accuracy & & & \\
Overall & .829 & .899 & .071 \\
Block 1 & .889 & .915 & .026 \\
Block 2 & .810 & .926 & .116 \\
Block 3 & .788 & .857 & .069 \\
\hline
\end{tabular}

first block $[t(20)=2.22, d=0.69]$ to $15 \mathrm{msec}$ in the second block $[t(20)=0.90, d=0.28]$ to $-7 \mathrm{msec}$ in the final block $[t(20)=-0.44]$. The final RT consistency advantage varied from $46 \mathrm{msec}$ in the first block $[t(20)=1.11, d=$ $0.34]$ to $-4 \mathrm{msec}$ in the second block $[t(20)=-0.11]$ to $13 \mathrm{msec}$ in the final block $[t(20)=0.35, d=0.11]$.

Overall, there was a strong consistency effect for old items in the accuracy data and a nonsignificant consistency advantage in RT, thus no speed-accuracy trade-off. This consistency effect suggests that the classification action was incorporated into the object representation.

\section{EXPERIMENT 2}

In Experiment 1, the locations of the bins were confounded with the action performed on an object, so the consistency advantage might have resulted from representing the action or from representing the ending location of the action. Experiment 2 examined this issue by changing the actions required in the classification phase. First, the classification bins were moved to the top and bottom of the front wall. Second, barriers were positioned to prevent participants from moving the objects directly up or down; they had to first move the object to the left or right, then move it in a slanted direction up or down. The recognition test phase was exactly the same as in Experiment 1. Consequently, the recognition old/new response was either the same as just the initial part of the classification judgment (consistent) or not (inconsistent). If the effect in Experiment 1 resulted at least in part from action consistency, Experiment 2 should produce a consistency advantage.

\section{Method}

Participants. Twenty-seven undergraduates participated. Data were excluded from 5 participants due to system failure (1) or duration of classification learning (4), leaving data from 22 participants.

Design, Materials, and Procedure. The only two changes from Experiment 1 were in classification learning. First, the two category bins were placed at the top and bottom of the center of the front wall. Second, virtual obstacles prevented participants from simply moving the item up or down to classify so that the initial action of the classification could be used for the consistency manipulation in recognition. In particular, a barrier (indicated by a straight line) appeared $30 \mathrm{~cm}$ above and below the fixation point, leaving a $60-\mathrm{cm}$ opening at one end but covering the rest of that wall and the adjacent wall. The top and bottom barriers left openings on different sides (Figure 1). Thus, to put an item in the top bin (Category A), participants had to move it to the left (as in Experiment 1 and for "old" items at test), then move at a slant up-right to the bin. Category $\mathrm{B}$ items required a right movement, then a slanted left-down movement.

\section{Results and Discussion}

Measures and analyses were the same as in Experiment 1 , including the trimming (3.2\% of initial RT, $3.8 \%$ of final RT).

Classification learning. The 22 participants varied in the number of 18 -item blocks to reach the classification learning criterion, 3 to $13(M=5.7, S D=2.6)$.

Recognition test. Recognition performance showed good old/new discriminability, with overall accuracy of .836 for initial actions (.809 hits, .863 correct rejections) and .886 for final actions (.875 hits, .897 correct rejections). Experiment 2 also showed a consistency advantage, but mainly for response latency to initiate an action (see Table 2). For initial RT, items with consistent actions across the learning and test phases were $34 \mathrm{msec}$ faster $[t(21)=2.54, d=0.77] ; 17$ of 22 participants showed this advantage. Items with consistent actions were faster

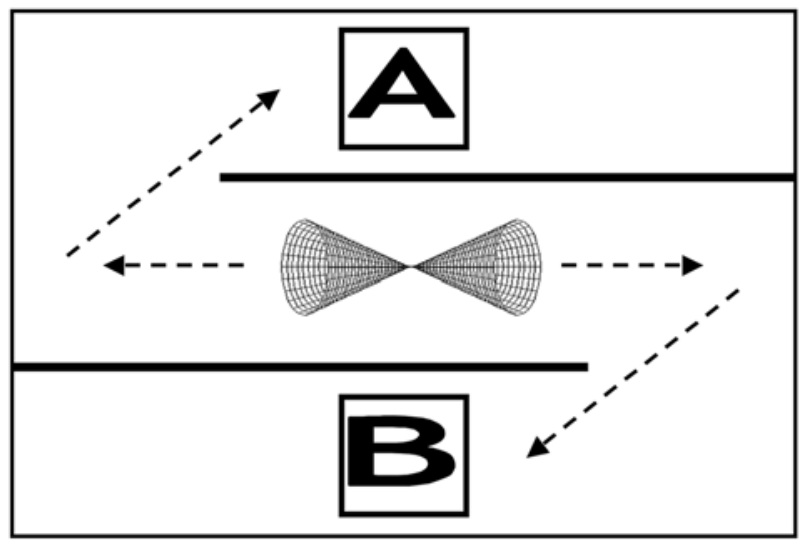

Figure 1. Schematic of obstacles, bins, and classification response paths for Experiment 2. A barrier (indicated by a solid line) appeared $30 \mathrm{~cm}$ above and below the fixation point. The top line began on the front wall $60 \mathrm{~cm}$ from the left side, continued right across the front wall and also covered the right wall. The bottom line had the reverse configuration, leaving an opening on the right side of the front wall. The paths to classify the items are in dashed lines. To put an item in the top bin (Category A), participants had to move it to the left then move at a slant upward to the right to the bin. Category $B$ items required a right movement, then a slanted left and downward movement. 
Table 2

Experiment 2: Mean Response Times (RTs, in Milliseconds) and Proportions Correct for Initial and Final Responses for "Old" Items

\begin{tabular}{crcc}
\hline & Inconsistent & Consistent & $\begin{array}{c}\text { Consistent } \\
\text { Advantage }\end{array}$ \\
\hline Initial RT & & & \\
Overall & 962 & 928 & 34 \\
Block 1 & 1,002 & 966 & 37 \\
Block 2 & 966 & 925 & 41 \\
Block 3 & 917 & 885 & 32 \\
Initial Accuracy & & & \\
Overall & .795 & .823 & .028 \\
Block 1 & .803 & .848 & .045 \\
Block 2 & .818 & .839 & .021 \\
Block 3 & .763 & .783 & .020 \\
Final RT & & & \\
Overall & 1,269 & 1,239 & 30 \\
Block 1 & 1,321 & 1,303 & 18 \\
Block 2 & 1,238 & 1,226 & 12 \\
Block 3 & 1,233 & 1,187 & 46 \\
Final Accuracy & & & \\
Overall & .870 & .880 & .010 \\
Block 1 & .874 & .904 & .030 \\
Block 2 & .874 & .889 & .025 \\
Block 3 & .864 & .848 & -.015 \\
\hline
\end{tabular}

in each block (with consistency effects of 37,41 , and $32 \mathrm{msec}$, respectively), but the difference was not always reliable $[t(21)=1.87$ and 1.06 for Blocks 1 and $3, d \mathrm{~s}=$ 0.56 and $0.32 ; t(20)=2.03$ for Block $2, d=0.63$, with the reduced $d f$ s due to an excluded participant who had no correct inconsistent responses in that block]. The consistency advantage was smaller and more variable for the final action RT $[M=30 \mathrm{msec}, t(21)=1.37, d=0.41]$. All three blocks showed small, nonsignificant consistency advantages $(18,12$, and $46 \mathrm{msec}$, with corresponding $t \mathrm{~s}$ of $0.70,0.43$, and 1.20 and corresponding $d \mathrm{~s}$ of $0.21,0.13$, and 0.36 ). Accuracy data showed only a small and nonsignificant consistency advantage. Accuracy of the initial action was .028 higher for items with a consistent action $[t(21)=0.89, d=0.27]$, and the final action accuracy was only .010 higher $[t(21)=0.31, d=0.09]$.

As can be seen in Table 2 (and to a lesser extent in Table 1), the consistency effect and recognition performance both show hints of decreasing over blocks. The consistency effect is expected to decrease as inconsistent items begin to get associated to their new actions. The drop in overall recognition performance is probably due to the greater difficulty in discrimination as new colors, shapes, and patterns are repeated in Blocks 2 and 3.

In sum, even when location and action are not confounded, there is a consistency advantage, clearest in the initial RT data. The final RT and accuracy differences were in the same direction but were not statistically significant. It is likely that the consistency effect might be larger if the classification and recognition actions were identical and more distinctive.

\section{GENERAL DISCUSSION}

The arbitrary action used when learning to classify an object is incorporated into its representation and affects later judgments about that object. Recognition judgments for old items were more accurate or faster if they required a response that was similar to the action used in classifying that object.

This result extends earlier findings in important ways. Research on action effects has concentrated on welllearned objects and affordances, whereas the current studies show effects from early learning of arbitrary actions. Research results on interaction effects during category learning (Love, 2003; Ross, 1996) may have been due to increased attention to particular features or relations, whereas the current studies suggest that interactions may also lead to specific action components' being incorporated. Actions are associated with an object, and those actions are activated when the object is recognized, perhaps as a result of some preparation to act.

Three aspects of our approach suggest an even greater influence of actions than that uncovered in other contexts. First, we examined the learning of new categories rather than using familiar objects, and we found influences of action even when training was limited. It was not obvious from prior work on actions and representations that such effects would be forthcoming with such a minimal amount of learning. Second, by using arbitrary actions rather than actions afforded by the objects, we can better determine whether or not the actions are truly incorporated into the object representation. If the actions are afforded by the objects themselves, participants need not represent those actions; they can simply perceive the same affordances whenever they see the object. These results suggest that affordances are not crucial for obtaining these later effects. Finally, the actions we used were not integral to the classification or recognition decision; rather, they were simply the response made on the basis of that decision. One might expect that actions integral to the decision would be more likely to be incorporated.

This research is a preliminary investigation, and we acknowledge that alternative explanations exist. For example, the representation might be directly associated not with the action, but rather with the directed motion. ${ }^{2}$ Further research could test this idea by having the action without the resulting object motion.

\section{Object Representations or Category Representations?}

We have discussed the findings as an indication that the object representations incorporate action information, but one might ask whether it is the category-level representations that incorporate this information. We can address this issue in a preliminary fashion by examining the influence of action on "new" items. In particular, "new" items with the classification learning features for color and shape but with new patterns (which were irrelevant to the classification) were still classifiable by any color-shape rule the participants had learned. If these new items show a consistency advantage, the action information is presumably represented at the category level. There is no evidence of a consistency effect in these data (or if one looks at all the "new" items). In Experiment 1, the inconsistent responses 
show a slight advantage for accuracy and RT (.032 and .011 for initial and final accuracy; $26 \mathrm{msec}$ and $34 \mathrm{msec}$ for initial and final RT). In Experiment 2, there is a consistency effect, but it is a bit smaller than the reverse one in Experiment 1 (.030 and .010; 15 and $27 \mathrm{msec})$. Thus, the consistency advantage appears to reflect the incorporation of action information into individual object representations. However, this conclusion should be greeted with caution, given that the experiments were not designed to distinguish these possibilities. The number of observations in some cells is small, and a consistency advantage for "new" items would likely be smaller than for "old" items, so it might be difficult for us to detect.

\section{Goal-Relevance of Action}

One final question is whether the facilitation of the action requires the action to be relevant to the goal for which the object is being used. In these experiments, the action was the classification response, though it was not involved in the classification decision itself. Future studies could examine this issue by not having the action during classification be a goal-directed part of the classification response. For example, an arrow at the fixation point could signal the learner to move the gamepad left or right, and only then would the object appear. The classification response would be a different action (e.g., buttonpress). This would still lead to the consistency effect for the left or right movement if the effect is the result of the physical action being temporally associated to the object, but not if the goal-relevance of the action to the object is critical.

\section{AUTHOR NOTE}

This work was supported by the Beckman Institute and by NSF Grant BCS 03-17681 to R. F. W. We thank the Beckman Integrated Systems Lab for help in conducting the experiment, Samuel Liu for testing the participants, and Blair Flicker for help in data analysis. Correspondence concerning this article should be addressed to B. H. Ross, Beckman Institute, University of Illinois, 405 N. Matthews Ave., Urbana, IL 61801 (e-mail: bhross@uiuc.edu).

\section{REFERENCES}

Barsalou, L. W. (1999). Perceptual symbol systems. Behavioral \& Brain Sciences, 22, 577-660.

BARSALOU, L. W. (2003). Situated simulation in the human conceptual system. Language \& Cognitive Processes, 18, 513-562.

Chainay, H., \& Humphreys, G. W. (2002). Privileged access to ac- tion for objects relative to words. Psychonomic Bulletin \& Review, 9, 348-355.

COHEN, J. (1988). Statistical power analysis for the behavioral sciences (2nd ed.). Hillsdale, NJ: Erlbaum.

Creem, S. H., \& Proffitt, D. R. (2001). Grasping objects by their handles: A necessary interaction between cognition and action. Journal of Experimental Psychology: Human Perception \& Performance, 27, 218-228.

Glenberg, A. M., \& KaschaK, M. P. (2002). Grounding language in action. Psychonomic Bulletin \& Review, 9, 558-565.

GrÈzes, J., \& DeCETY, J. (2001). Functional anatomy of execution, mental simulation, observation, and verb generation of actions: A metaanalysis. Human Brain Mapping, 12, 1-19.

Love, B. C. (2003). The multifaceted nature of unsupervised category learning. Psychonomic Bulletin \& Review, 10, 190-197.

Markman, A. B., \& Ross, B. H. (2003). Category use and category learning. Psychological Bulletin, 129, 592-613.

Pavese, A., \& Buxbaum, L. J. (2002). Action matters: The role of action plans and object affordances in selection for action. Visual Cognition, 9, 559-590.

Pecher, D., \& ZwaAn, R. A. (EDs.) (2005). Grounding cognition: The role of perception and action in memory, language, and thinking. Cambridge: Cambridge University Press.

Ross, B. H. (1996). Category representations and the effects of interacting with instances. Journal of Experimental Psychology: Learning, Memory, \& Cognition, 22, 1249-1265.

Ross, B. H., Chin-Parker, S., \& Diaz, M. (2005). Beyond classification learning: A broader view of category learning and category use. In W. Ahn, R. L. Goldstone, B. C. Love, A. B. Markman, \& P. Wolff (Eds.), Categorization inside and outside the laboratory: Essays in honor of Douglas L. Medin (pp. 197-213). Washington, DC: APA.

Sмiтн, L. B. (2005). Action alters shape categories. Cognitive Science, 29, 665-679.

TuCKER, M., \& ElLis, R. (2001). The potentiation of grasp types during visual object categorization. Visual Cognition, 8, 769-800.

\section{NOTES}

1. The materials were selected based on a norming study. Items were shown in a same/different task with a 2-sec presentation of the first item and a 1-sec delay before the second item. The "different" items were different on one feature (color, shape, or pattern). There were 10 colors, 12 shapes, and 10 patterns. Each of 6 participants responded to 256 trials (156 different trials and 100 same trials). The different trials included all possible contrasts of values of a feature, though the other two features remained constant. Thus, for the 10 colors, we included all 45 pairs of different colors, but each of those 45 different trials had the same shape and pattern for the two objects, though these shapes and patterns were varied over the 45 pairs. We chose feature values for the experiment that were easily discriminable (i.e., fast "different" responses when the feature was changed).

2 . We thank a reviewer for this suggestion.

(Manuscript received January 21, 2006; revision accepted for publication July 25, 2006.) 